\title{
Place Physical Address
}

National Cancer Institute

\section{Source}

National Cancer Institute. Place Physical Address. NCI Thesaurus. Code C94274.

A representation of the location of the place. 